\title{
Sistema de Classificação Automática de Carcaças Bovinas
}

\author{
Carmen Dalla Rosa Bittencourt ${ }^{1}$, Marcelo Ladeira ${ }^{1}$, Saul Fontoura da Silva ${ }^{2}$, \\ Anderson Luis S. Bittencourt, Díbio Leandro Borges ${ }^{1}$ \\ ${ }^{1}$ Departmento de Ciência da Computação - Universidade de Brasília \\ ${ }^{2}$ Departamento de Medicina Veterinária Preventiva - Universidade Federal de Santa \\ Maria (UFSM) - Santa Maria, RS - Brasil \\ carmendr@terra.com.br, mladeira@unb.br, saul@smail.ufsm.br, abitten@bol.com.br, dibio@unb.br
}

\begin{abstract}
The Brazilian bovine carcass classification process is regulated by the Brazilian Agriculture Ministry decree number 612 of December 05, 1989. Although the usual bovine carcass classification process is carried out by a qualified technician it is very subjective because it is based on a visual inspection of predefined carcass areas during the bovine abate. This paper introduces a novel approach to automate the classification of bovine carcass based on neural-network image segmentation to estimate the bovine carcass fatness amount.
\end{abstract}

Resumo. No Brasil, a classificação do acabamento de gordura de carcaças bovinas é regulamentado pela Portaria de n. 612 de 05/12/1989 do Ministério da Agricultura. Esse processo é realizado por meio da observação visual e subjetiva através da análise de regiões predefinidas, feita por um profissional habilitado, durante o processo de abate nas indústrias frigoríficas. Esse artigo propõe o uso de redes neurais artificiais para, de forma automática, segmentar e classificar o acabamento de gordura de carcaças bovinas em imagens digitais.

\section{Introdução}

A tipificação de carcaças é um instrumento auxiliar na comercialização de gado e carne que deve ter surgido no fim do século 19 ou início do século 20, e que ainda hoje é comumente utilizado em países, como Brasil, Estados Unidos, Canadá, Argentina, Uruguai, entre outros. A tipificação é formada de duas partes, sendo a primeira de classificação dos lotes por sexo, pela maturidade e pela faixa de peso do gado, de modo que as carcaças são agrupadas por categoria. A segunda parte é a tipificação propriamente dita, que consiste em alocar as carcaças das principais categorias, como novilho ou novilha, em tipos ordenados de melhor a pior, segundo outros indicadores tradicionalmente utilizados nos julgamentos de gado de corte em exposições, como a conformação e a quantidade de gordura (acabamento). Em tese as carcaças dos melhores tipos dariam carne de melhor qualidade [FELÍCIO, 2005].

Desde os primeiros estudos sistemáticos das normas de classificação e tipificação de carcaças no final da década de 60 há o interesse manifesto de se implantar um sistema de classificação prático, eficiente, dinâmico e universal, a ser utilizado pelos países importadores e exportadores. No Brasil, os esforços para definir uma metodologia para classificação e tipificação de carcaças bovinas resultaram na 
publicação da Portaria Ministerial no 612/89, do Ministério da Agricultura, Pecuária e Abastecimento (MAPA), que instituiu o Sistema Nacional de Tipificação de Carcaças Bovinas. Essa Portaria determina que a tipificação de carcaças deve se basear nos parâmetros sexo-maturidade, conformação, acabamento e peso. A carcaça bovina é definida como sendo o animal abatido, sangrado, esfolado, eviscerado, desprovido de cabeça, patas, rabada, glândulas mamárias na fêmea, ou verga, exceto suas raízes, e testículos, no macho. Após a divisão em meia carcaça (Figura 1), retiram-se ainda os rins, gorduras perirrenal e inguinal, "ferida de sangria", medula espinhal, diafragma e seus pilares.
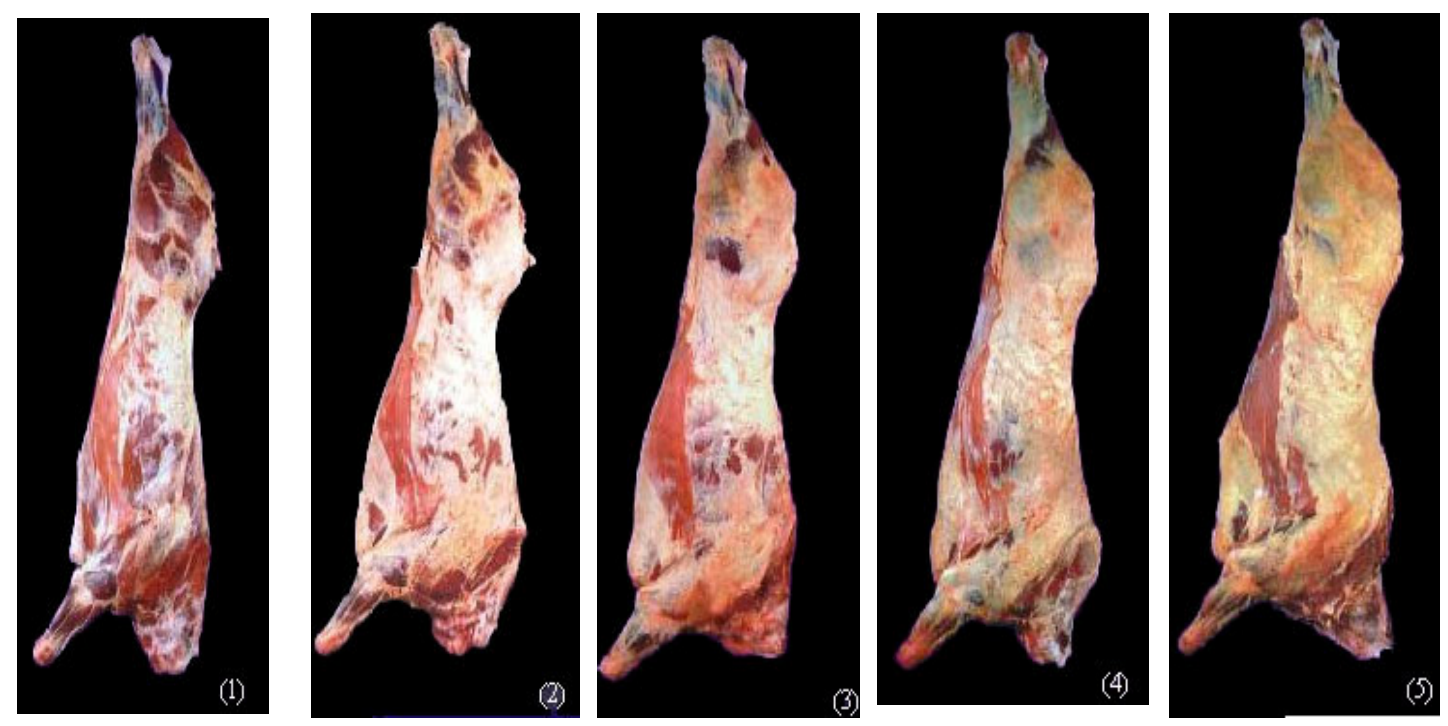

Figura 1 - Classificações de Acabamento de Gordura

Como o objetivo deste artigo é apresentar um sistema para classificação automática do acabamento de gordura, somente este parâmetro será descrito de forma mais detalhada. A classificação de acabamento expressa a distribuição e a quantidade de gordura de cobertura da carcaça, sendo descrita através das categorias na Tabela 1.

Tabela 1 - Categorias de acabamento de gordura

\begin{tabular}{|c|c|}
\hline Categoria & Espessura de Gordura \\
\hline Magra (1) & Gordura ausente \\
\hline Gordura escassa (2) & Gordura de $1 \mathrm{~mm}$ a $3 \mathrm{~mm}$ de espessura \\
\hline Gordura mediana (3) & Gordura acima de $3 \mathrm{~mm}$ e até $6 \mathrm{~mm}$ de espessura \\
\hline Gordura uniforme (4) & Gordura acima de $6 \mathrm{~mm}$ e até $10 \mathrm{~mm}$ de espessura \\
\hline Gordura excessiva (5) & Gordura acima de $10 \mathrm{~mm}$ de espessura \\
\hline
\end{tabular}

Fonte: Portaria 612/89 do Ministério da Agricultura

\subsection{Descrição do problema}

A aferição da gordura é feita em três locais diferentes da carcaça, a saber: altura da $6^{\mathbf{a}}$ costela, sobre o músculo grande dorsal (em sua parte dorsal), altura da $9^{\mathrm{a}}$ costela, sobre 
o músculo grande dorsal (em sua parte ventral) e altura da $12^{\mathrm{a}}$ costela, sobre o músculo serrátil dorsal caudal. Complementarmente proceder-se-á a verificação da gordura na região lombar e no coxão. A avaliação da carcaça e seu enquadramento dentro de um determinado tipo, é realizada a quente depois da pesagem e antes de entrar na câmara de resfriamento. Essa avaliação e a posteriormente classificação do acabamento é realizada através de observação visual da distribuição da gordura sobre a superfície da carcaça, por um técnico habilitado pelo MAPA. Embora guiado por procedimentos prédeterminados, por se tratar de um processo subjetivo [FELÍCIO, 2005] não se pode garantir ausência de erros e nem mesmo padronização na classificação, isto é que os técnicos irão atribuir a mesma classificação para a mesma carcaça bovina.

A literatura especializada mundial cita diversos sistemas de classificação de acabamento de gordura. Em sua grande maioria, a classificação é feita por um técnico habilitado, por meio da análise visual da gordura intramuscular (denominada marmorização), utilizando uma amostra da carcaça resfriada, geralmente retirada entre a $6^{\mathrm{a}}$ e a $7^{\mathrm{a}}$ ou entre a $12^{\mathrm{a}}$ e $13^{\mathrm{a}}$ costelas. Essa abordagem é utilizada na Austrália [SAINZ \& ARAUJO, 2001], Estados Unidos [SAINZ \& ARAUJO, 2001] Uruguai [BRITO, et al. 2006] e Argentina [BARCELO \& PEREYRA, 2005]. Existe também um sistema de solução proprietária, que faz análise em imagem 3D de cada metade da carcaça, mas o que é analisado é o peso do animal abatido, e não o acabamento de gordura.

Com o intuito de desenvolver métodos objetivos de tipificação, capazes de funcionar com alta velocidade, acurácia e precisão, e a um custo acessível, várias tecnologias tem sido estudadas. Estas se resumem nas que simulam a observação visual do avaliador, como é o caso desta pesquisa, e naquelas que medem alguma característica não observável visualmente. As primeiras incluem a ultra sonografia e a análise de imagens de vídeo ou foto digital, enquanto que a segunda categoria inclui a condutividade elétrica total e a impedância bioelétrica [SAINZ \& ARAUJO, 2001, p.21].

Pelos motivos acima mencionados se faz necessária, na atual situação do Brasil de maior exportador (em volume) de carne bovina do mundo, uma classificação bastante precisa. A classificação deve fornecer aos frigoríficos informações sobre o rendimento da carcaça, ao ponto de torná-los aptos a empregarem tabelas de pagamento diferenciados aos produtores de animais de mais alta qualidade e que apresentem um melhor rendimento (tipos 3, 4 e 5).

\section{Abordagem da Solução Proposta}

O método proposto se divide basicamente em duas etapas. A primeira delas compreende a criação de uma rede neural do tipo MLP (perceptron de múltiplas camadas) capaz de segmentar a área correspondente a gordura em imagens digitais de carcaças bovinas. A segunda etapa corresponde a tarefa de criação de uma rede neural, também do tipo MLP, capaz de classificar a imagem resultante da primeira etapa de acordo com os cinco padrões de acabamento de gordura descritos anteriormente.

As imagens utilizadas foram obtidas durante o processo de abate, no padrão de cores RGB e gravadas no padrão de compressão JPG, com a resolução espacial de 1600 x 600 pixels. 


\subsection{Segmentação da área de gordura}

Nesta etapa foi utilizada uma rede neural do tipo MLP, capaz de identificar as regiões de gordura em uma imagem digital da carcaça bovina. Esta segmentação baseou-se no fato de que os pixels correspondentes a área de gordura possuem um padrão característico com relação a intensidade de cor nos elementos $\mathrm{R}, \mathrm{G}$ e $\mathrm{B}$ da imagem digital colorida. Para uma maior efetividade e rapidez no treinamento, foi realizada a normalização de todos os dados de entrada, no intervalo de 0 a 1 . Foram utilizados dois conjuntos de treinamento. O primeiro conjunto, denominado $\mathrm{T} 1 \mathrm{Cj} 1$, foi composto de 6.668 amostras de pixels de gordura e 13.332 amostras de pixels de não-gordura, totalizando $20 \mathrm{mil}$ amostras, formando uma matriz $3 \times 20.000$, onde as linhas corresponde ao padrão de cor RGB e as colunas correspondem às amostras disponíveis no conjunto de treinamento. Analogamente, o segundo conjunto, T1Cj2, foi composto de 13.336 amostras de pixels de gordura e 26.664 amostras de pixels de não-gordura, totalizando 40.000 amostras, formando uma matriz $3 \times 40.000$.

Para cada um dos conjuntos de entrada acima foi criado um vetor de saída correspondente. O primeiro, correspondendo a $1 \times 20.000$ posições e o segundo, correspondendo a 1x40.000 posições. Ambos os conjuntos possuem valores 1 para pixels correspondentes a gordura e 0 para pixels classificados como não gordura nos conjuntos de treinamento.

A definição da rede neural mais apropriada a determinado problema é essencialmente empírica [HAYKIN, 2001]. Para avaliar a capacidade de segmentação da área correspondente a gordura da imagem digital da carcaça bovina foram propostas quatro topologias de redes do tipo MLP descritas a seguir. Como função de ativação, foi utilizada a função logarítmica sigmóide em todas as redes propostas.

Rede 1 (Net321LM): contém 3 neurônios na camada de entrada, 2 neurônios na camada intermediária e um neurônio na camada de saída.

Rede 2 (Net371LM): contém 3 neurônios na camada de entrada, 7 neurônios na camada intermediária e um neurônio na camada de saída.

Rede 3 (Net381LM): contém 3 neurônios na camada de entrada, 8 neurônios na camada intermediária e um neurônio na camada de saída.

Rede 4 (Net391LM): contém 3 neurônios na camada de entrada, 9 neurônios na camada intermediária e um neurônio na camada de saída.

Para realizar o treinamento das redes neurais descritas acima, foi utilizado o método de treinamento Levenberg-Marquardt [HAGAN \& MENHAJ, 1994].

$\mathrm{O}$ processo de treinamento de cada uma das redes ocorreu em duas etapas. Inicialmente a rede foi treinada utilizando-se o conjunto de treinamento $\mathrm{T} 1 \mathrm{Cj} 1 \mathrm{e}$ seu respectivo vetor de saída. Em seguida, partindo da rede já treinada com o conjunto de treinamento $\mathrm{T} 1 \mathrm{Cj} 1$, realizou-se novamente o treinamento utilizando-se o conjunto de treinamento $\mathrm{T} 1 \mathrm{Cj} 2$ e seu respectivo vetor de saída.

Exemplos do resultado do processo de segmentação das imagens, podem ser observados na Figura 2. 


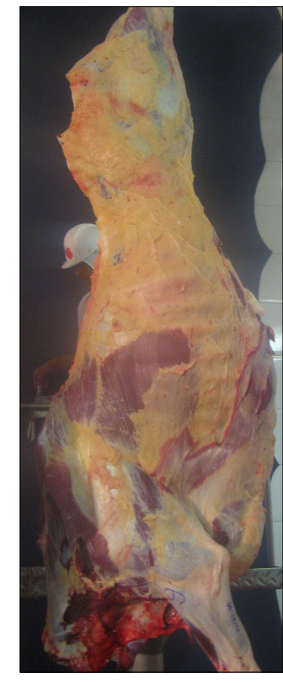

Segmentação
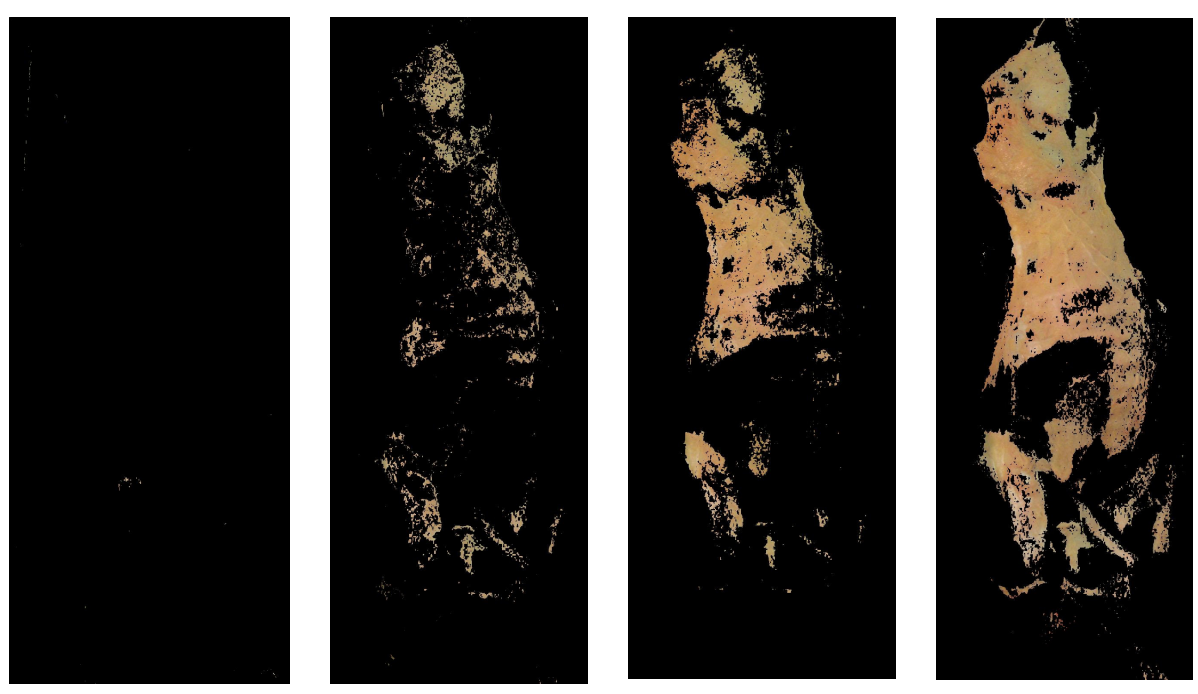

Rede 1: Net321LM

Rede 2: Net371LM Rede 3: Net381LM

Rede 4: Net391LM

\subsection{Classificação de acabamento}

Nesta etapa foi utilizada uma rede neural do tipo MLP, capaz de classificar as regiões de gordura segmentadas da imagem digital, de acordo com as 5 categorias apresentadas na Tabela 1. Esta segmentação baseia-se na classificação do padrão de distribuição do acabamento de gordura da carcaça na imagem digital segmentada. Se essa classificação for executada diretamente a partir da imagem segmentada teremos 1600 x 600, ou seja 960.000 neurônios na camada de entrada rede neural de classificação.

Com o objetivo de reduzir o número de entradas na rede neural de classificação do acabamento da gordura da carcaça bovina, foi realizado um pré-processamento onde cada imagem foi transformada em uma matriz de 32x12. O valor de cada posição desta matriz é calculado com base na quantidade de pixels de gordura existente em uma máscara de 50x50 pixels da imagem segmentada. Caso a quantidade de pixels de gordura na máscara da imagem segmentada seja maior que a metade dos pixels existentes na mesma máscara, a posição da matriz recebe valor 1 , caso contrário recebe valor 0. Cada posição desta nova matriz serve de entrada para a rede neural utilizada para realizar a classificação da distribuição da gordura de acabamento, totalizando 384 entradas.

A Figura 3 apresenta exemplos de acabamento de gordura, dos tipos 1 a 4, com suas respectivas imagens segmentadas, imagens após aplicação da máscara 50x50 pixels e as respectivas matrizes de entrada - para a rede de classificação - geradas.

Para o treinamento da rede neural de classificação foi utilizado um conjunto de treinamento denominado T2Cj1 (Tabela 2). Tal conjunto de treinamento é composto de 05 (cinco) imagens segmentadas do tipo 1, 26 (vinte e seis) imagens segmentadas do tipo 2, 123 (cento e vinte e três) imagens segmentadas do tipo 3 e 3 (três) imagens segmentadas do tipo 4, correspondendo a um total de 157 amostras. Essas quantias reproduzem a distribuição de tipos de carcaças bovinas no Brasil, segundo informação verbal do MAPA. Nenhuma imagem do tipo 5 foi coletada nos frigoríficos pesquisados, portanto, é oportuno ressaltar que não foram consideradas nessa pesquisa por ocorrerem 
com frequência muito baixa. Um dos fatores que explicam essa baixa ocorrência é o fato de ser antieconômico para o produtor engordar em demasia o seu gado bovino.

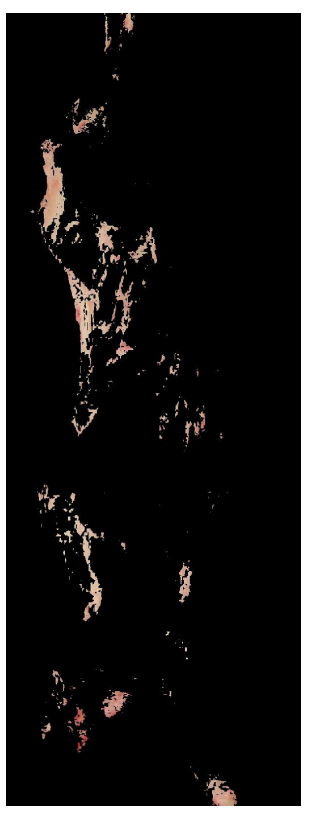

Acabamento

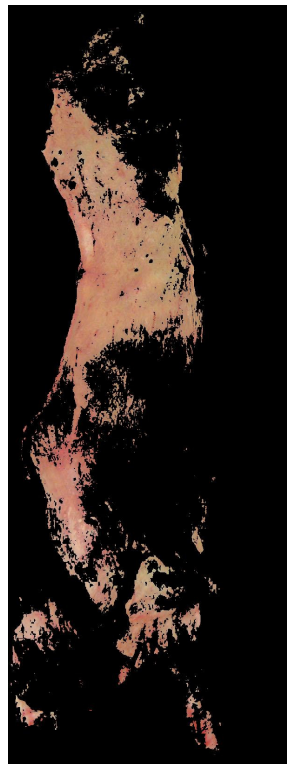

Acabamento

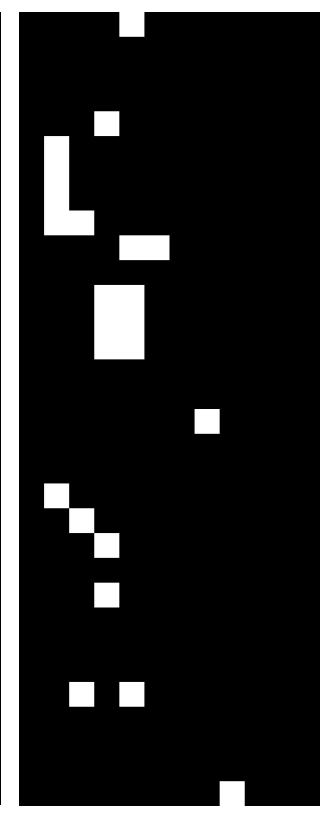

tipo 1

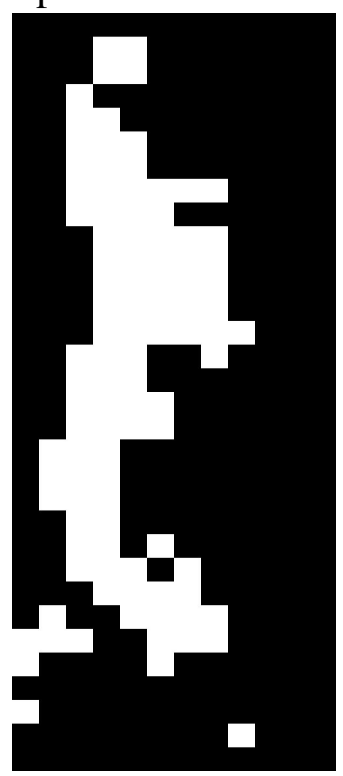

tipo 3
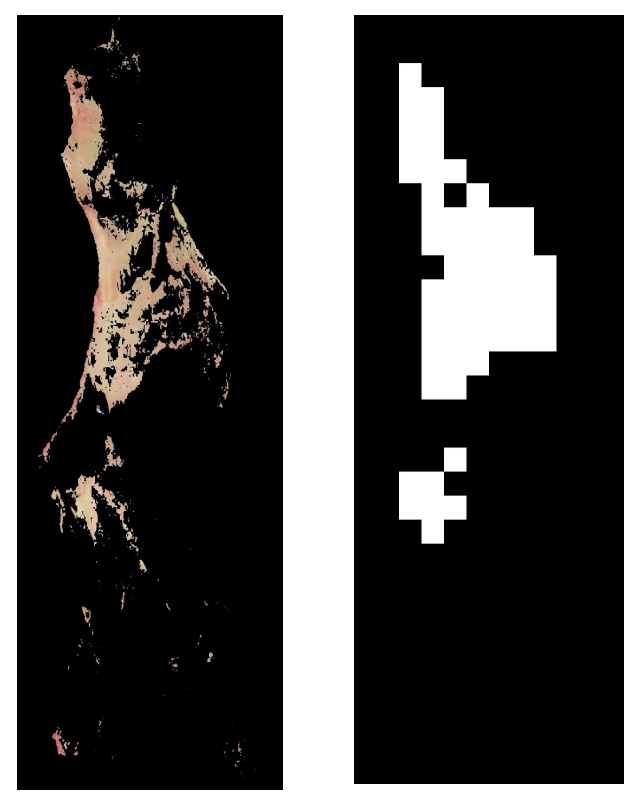

Acabamento
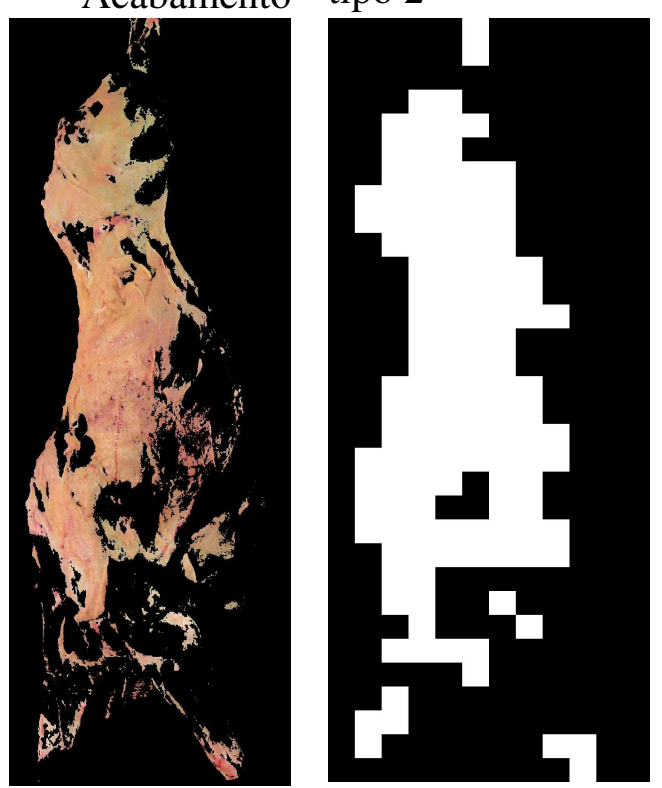

Acabamento

Figura 3 - Representação do Pré-processamento em Imagens de Tipos 1 a 4

Cada uma das imagens utilizadas foi submetida ao pré-processamento com máscara de 50x50 pixels, antes de ser incluída no conjunto de treinamento. O total de amostras utilizados no conjunto de treinamento é representado por uma matriz de $384 \times 157$ onde cada coluna representa informações relativas a uma imagem da amostra. Para essas variáveis de entrada corresponde a matriz de saída denominada T2Cj1A. Tal matriz possui dimensões $4 \times 157$ onde cada coluna possui o resultado esperado para cada amostra correspondente no conjunto de treinamento $\mathrm{T} 2 \mathrm{Cj} 1$. 
Tabela 2 - Conjunto de Treinamento: Classificação do Acabamento de Gordura

\begin{tabular}{|c|c|c|c|c|c|c|}
\hline Conjunto & Tipo 1 & Tipo 2 & Tipo 3 & Tipo 4 & Total & Saída \\
\hline T2Cj1 & 5 & 26 & 123 & 3 & 157 & T2Cj1A \\
\hline
\end{tabular}

A rede neural para classificação utilizada era do tipo MLP com 384 neurônios na camada de entrada, 24 na camada escondida e 4 na camada de saída. Cada neurônio na camada de entrada, corresponde a uma posição da matriz gerada no pré-processamento da imagem segmentada e cada neurônio na camada de saída, corresponde a um tipo de classificação de acabamento de gordura possível. Como função de ativação, foi usada a função de transferência logarítmica sigmóide em todas as camadas da rede. A rede foi treinada com o método backpropagation [RUMELHART, McCLELLAND, 1986].

$\mathrm{O}$ processo de treinamento da rede ocorreu em uma etapa. A rede foi treinada utilizando-se o conjunto de treinamento $\mathrm{T} 2 \mathrm{Cj} 1$ e sua respectiva matriz de saída. Essa rede neural foi denotada Net384_24_4.

\section{Resultados Obtidos}

Para avaliação dos resultados obtidos pela rede treinada anteriormente, foram realizadas simulações com um conjunto de teste. Tal conjunto, denominado T2Cj1T contém informações referentes a 05 (cinco) imagens do tipo 1, 26 (vinte e seis) imagens do tipo 2, 123 (cento e vinte três) imagens do tipo 3 e 3 (três) imagens do tipo 4, correspondendo a um total de 157 amostras, como no caso do conjunto de treinamento.

Por se tratar de classificadores, para a discriminação entre os modelos gerados foram utilizados índices obtidos através da matriz de confusão. Para classificação multiclasses, as classificações no conjunto de avaliação são apresentadas na forma de uma matriz bidimensional com uma linha e uma coluna para cada classe. Cada elemento desta matriz, chamada matriz de confusão, apresenta o número de casos avaliados na qual a classe real $(\mathrm{R})$ é a linha e a classe predita $(\mathrm{P})$ pelo classificador é a coluna. Bons resultados são caracterizados por valores altos na diagonal principal e valores nulos para elementos fora da diagonal principal. Suponha que um classificador está sendo utilizado para classificar uma instância que possui apenas duas classes, por exemplo, "sim" e "não". A Tabela 3 ilustra a nomenclatura utilizada para as classificações possíveis.

Tabela 3. Matriz de Confusão com duas Classes

\begin{tabular}{|c|l|l|}
\hline $\mathbf{R} \backslash \mathbf{P}$ & \multicolumn{1}{|c|}{ sim } & \multicolumn{1}{c|}{ não } \\
\hline sim & Verdadeiro Positivo (VP) & Falso Negativo (FN) \\
\hline não & Falso Positivo (FP) & Verdadeiro Negativo (VN) \\
\hline
\end{tabular}

Nessa análise foram utilizados os índices apresentados na Tabela 4. Todos eles assumem valores no intervalo [0,1] e podem ser calculados a partir da matriz de confusão.

Tabela 4. Índices para Discriminação entre Classificadores Dicotômicos

\begin{tabular}{|c|c|c|c|}
\hline Sensibilidade $(\mathbf{S})$ & Especificidade $(\mathbf{E})$ & Acurácia $\left(\mathbf{A}_{\mathbf{c}}\right)$ & $\mathbf{F}_{\mathbf{1}}$ \\
\hline $\mathrm{S}=\mathrm{VP} /(\mathrm{VP}+\mathrm{FN})$ & $\mathrm{E}=\mathrm{VN} /(\mathrm{VN}+\mathrm{FP})$ & $\begin{array}{c}\mathrm{A}_{\mathrm{c}}=(\mathrm{VP}+\mathrm{VN}) / \\
(\mathrm{VP}+\mathrm{FP}+\mathrm{VN}+\mathrm{FN})\end{array}$ & $\begin{array}{c}\mathrm{F}_{1}=2 * \mathrm{~S} * \mathrm{E} \\
/(\mathrm{S}+\mathrm{E})\end{array}$ \\
\hline
\end{tabular}


A sensibilidade é a taxa de verdadeiro positivo (TVp); a especificidade é a taxa de verdadeiro negativo (TVn) e a acurácia é a probabilidade de acerto do classificador. $\mathrm{O}$ melhor classificador é o que possui maior acurácia. $\mathrm{F}_{1}$ é uma média harmônica entre sensibilidade e especificidade. Por esse produto, o melhor classificador é aquele que maximiza a taxa de verdadeiro positivo e, simultaneamente, minimiza a taxa de falso positivo, ou seja o lixo associado aos casos selecionados como positivo. Note que o ponto de máximo para as métricas de acurácia e $\mathrm{F}_{1}$ ocorre quando VP e VN tendem para 1. A Tabela 5 apresenta o número de imagens corretamente classificadas pela rede neural Net384_24_4. Os resultados obtidos com segmentação da imagem original realizada pela rede neural com 9 neurônios na camada escondida, Net391LM, foram os com melhor desempenho na classificação.

Tabela 5 - Classificações Corretas Obtidas com a Rede Net384_24_4

\begin{tabular}{|c|c|c|c|c|}
\hline Categorias & $\mathbf{1}$ & $\mathbf{2}$ & $\mathbf{3}$ & $\mathbf{4}$ \\
\hline Imagens & $\mathbf{5}$ & $\mathbf{2 6}$ & $\mathbf{1 2 3}$ & $\mathbf{3}$ \\
\hline Net321LM & 5 & 0 & 0 & 0 \\
\hline Net371LM & 5 & 7 & 51 & 0 \\
\hline Net381LM & 5 & 9 & 56 & 0 \\
\hline Net391LM & 5 & 14 & 112 & 3 \\
\hline
\end{tabular}

A Tabela 6 apresenta a matriz de confusão obtida com a aplicação da rede Net391LM para segmentar as imagens originais e a rede Net384_24_4 para classificar o tipo da carcaça bovina. Os valores na diagonal principal representam as quantidades de carcaças que foram classificadas corretamente pela rede neural.

Tabela 6 - Matriz de Confusão Obtida com as Redes Net391LM e Net384_24_4

\begin{tabular}{|c|c|c|c|c|c|}
\hline $\mathbf{R} \backslash \mathbf{P}$ & $\mathbf{1}$ & $\mathbf{2}$ & $\mathbf{3}$ & $\mathbf{4}$ & Real \\
\hline $\mathbf{1}$ & 5 & 0 & 0 & 0 & 5 \\
\hline $\mathbf{2}$ & 8 & 14 & 4 & 0 & 26 \\
\hline $\mathbf{3}$ & 0 & 9 & 112 & 2 & 123 \\
\hline $\mathbf{4}$ & 0 & 0 & 0 & 3 & 3 \\
\hline Previsto & 13 & 23 & 116 & 5 & 157 \\
\hline
\end{tabular}

Os índices apresentados na Tabela 4 podem ser facilmente generalizados para classificadores n-classes a partir de uma análise de cada classe com a abordagem umcontra-todos. A Tabela 7 apresenta as matrizes de confusão geradas, a partir da Tabela 6 , com a abordagem de um-contra-todos.

Tabela 7 - Matriz de Confusão por Tipo de Carcaça

\begin{tabular}{|c|c|c|c|c|c|c|c|c|c|c|c|}
\hline $\mathbf{R} / \mathbf{P}$ & $\mathbf{1}$ & $\neg \mathbf{1}$ & $\mathbf{R} / \mathbf{P}$ & $\mathbf{2}$ & $\neg \mathbf{2}$ & $\mathbf{R} / \mathbf{P}$ & $\mathbf{3}$ & $\neg \mathbf{3}$ & $\mathbf{R} / \mathbf{P}$ & $\mathbf{4}$ & $\neg \mathbf{4}$ \\
\hline $\mathbf{1}$ & 5 & 0 & $\mathbf{2}$ & 14 & 12 & $\mathbf{3}$ & 112 & 11 & $\mathbf{4}$ & 3 & 0 \\
\hline$\neg \mathbf{1}$ & 8 & 144 & $\neg \mathbf{2}$ & 9 & 122 & $\neg \mathbf{3}$ & 4 & 30 & $\neg \mathbf{4}$ & 2 & 152 \\
\hline
\end{tabular}


A Tabela 8 apresenta os índices de desempenho obtido para cada tipo de carcaça bovina (valores de 1 a 4) e os índices médios na classificação obtida com as redes Net391LM e Net384_24_4.

Tabela 8. Performance Obtida com as Redes Net391LM e Net384_24_4

\begin{tabular}{|l|c|c|c|c|}
\hline \multicolumn{1}{|c|}{ Categoria } & Sensibilidade & Especificidade & Acurácia & F $_{\mathbf{1}}$ \\
\hline Magra (1) & 1 & 0.9474 & 0.9490 & 0.9630 \\
\hline Gordura escassa (2) & 0.5385 & 0.9313 & 0.8662 & 0.6824 \\
\hline Gordura mediana (3) & 0.9106 & 0.8824 & 0.9045 & 0.8962 \\
\hline Gordura uniforme (4) & 1 & 0.9870 & 0.9873 & 0.9936 \\
\hline Média esperada & 0.8535 & 0.8945 & 0.9011 & 0.8651 \\
\hline
\end{tabular}

A acurácia da classificação de carcaça, com a abordagem proposta é de $90 \%$, um valor animador mas que ainda precisa ser melhorado. Atualmente, essa pesquisa está na fase de desenvolvimento de equipamentos para permitir a coleta automática de imagens de carcaças bovinas durante o abate, in loco. Até o momento foram coletadas 2000 imagens, a maioria das categorias 2 e 3 . Com o aumento do número de imagens coletadas, espera-se poder treinar novas redes que apresentem desempenho superior ao obtido até o momento. Na análise apresentada, a classificação predita pelo sistema foi confrontada com a classificação realizada por um especialista humano, técnico habilitado pelo MAPA.

\section{Conclusão}

Esse artigo apresenta uma nova proposta para a classificação automática de carcaças bovinas. O levantamento bibliográfico realizado evidenciou poucos artigos publicados, sendo a pesquisa para classificação automática de carne mais explorada nas indústrias frigoríficas internacionais. Uma possível explicação para esse fato é que os resultados obtidos são implementados na forma de sistemas automatizados na própria indústria e tratados como segredo industrial. Não há, no Brasil, nenhuma indústria frigorífica dotada de sistema de classificação automática de carne. Essas características dificultam a comparação da proposta apresentada com outras abordagens existentes.

$\mathrm{O}$ tema abordado nesse artigo situa-se na fronteira do conhecimento $\mathrm{O}$ método ora proposto visa a implementação de um sistema, que irá disponibilizar, em tempo real, à indústria frigorífica a automatização do processo de classificação do acabamento de gordura. Este sistema avaliará de forma objetiva, o que o profissional do frigorífico hoje faz de forma subjetiva. Através desta automatização haverá uma maior transparência entre indústria frigorífica e pecuaristas, pois eliminará a subjetividade decorrente da análise visual humana, fornecendo ao pecuarista a certeza de que a carcaça de seu animal está sendo classificada por um sistema padronizado que não possui nenhuma subjetividade na análise. Neste contexto, ganha o pecuarista com o aumento da qualidade da classificação realizada e do valor agregado de seu produto, e ganha a pecuária brasileira que oferecerá um produto mais padronizado para o mercado externo. Por ser um sistema, o mesmo será passível de auditoria para comprovação da eficácia na classificação das carcaças. 
A maior limitação nesta pesquisa é que os resultados foram obtidos com um número pequeno de imagens, apenas 2000, sendo a maioria das categorias 2 e 3 . O número de carcaças das categorias 1 e 4 coletadas em campo foi extremamente baixo. Não foi coletada nenhuma carcaça da categoria 5 (gordura excessiva). É provável que a baixa freqüência de carcaças 1, 4 e 5 esteja associada ao sistema de engorda de boi adotado no Brasil. Nesse contexto, a acurácia de classificação obtida foi de $90 \%$, valor animador mas que ainda precisa ser melhorado. Com o aumento do número de imagens e a coleta de mais imagens das categorias menos frequientes, espera-se poder treinar novas redes que apresentem desempenho superior ao obtido até o momento.

Como trabalho futuro pretende-se utilizar técnicas de processamento de imagens através do uso de histograma de cores e análise de textura com a aplicação da autocorrelação da imagem.

\section{Referências}

Barceló, M.E., Pereyra, J. (2005) Recepción de Carne Vacuna: Reinspección y Registro. Dirección Nacional de Sanidad de las Fuerza Armadas. División Abastecimientos. Julio de 2005. Disponível em www.compraestatales.gub.uy/noticias_especiales/ archivos/Manual_recepcion_de_Carne_Vacuna.pdf (acesso em 25/10/2007)

Brito, G., Lima, J.M.S. de, Julián, R.S. \& Montossi, F. (2006) "Métodos para predecir el rendimiento carnicero de una canal vacuna". Programa Nacional de Producción de Carne y Lana. Revista INIA de Investigaciones Agronómicas, n.8, p.10-12.

Felício, P.E. de (2005) Classificação e Tipificação de Carcaças Bovinas. Palestra proferida no Congresso CBNA de 17-18 de maio de 2005, em Goiânia, Goiás. Disponível em http://www.fea.unicamp.br/deptos/dta/carnes/files/CTC_ITAL_06/ Classificacao_e_Tipificacao.pdf (Acesso em 25/10/2007, 12p.)

Hagan, M.T. \& Menhaj, M.B. (1994) "Training Feedforward Networks with the Marquardt Algorithm”. IEEE Transactions on Neural Networks, v.5, n.6., p.989-993,

Haykin, S. (2001) Redes Neurais: Princípios e Prática (Trad. Paulo Martins Engel). Porto Alegre: Bookman.

Rumelhart, D. E. and McClelland, J. L. (1986). Parallel Distributed Processing: Explorations in the Microstructure of Cognition, v.1. MIT Press, Cambridge, MA.

Sainz, R.D. \& Araujo, R.C. (2001) "Tipificação de Carcaças de Bovinos e Suinos". In: Anais do I Congresso Brasileiro de Ciência e Tecnologia de Carne, p.4-6 São Pedro, São Paulo. Disponível em http://www.fmvz.unesp.br/andrejorge/SAINZ_2001_ Tipificacao_Carcacas_Bovinos_Suinos.pdf (acesso em 26/10/2007) 